\title{
The Safe Use of Ultrasound in Medical Diagnosis
}

$3^{\text {rd }}$ edition 2012, edited by Gail ter Haar, BIR|Open; ISBN 978-0-905749-78-2

(eBook)

http://www.birjournals.org/site/books/ ultrasound.xhtml

Mittlerweile in seiner 3. Auflage ist dieses nun mehr online und kostenlos zur Verfügung stehende Buch eine aktuelle Wissensbilanz zur sicheren Anwendung der Ultraschalldiagnostik. In verschiedenen Kapiteln werden hier von namhaften Fachkollegen die wissenschaftliche Forschung und Ergebnisse wiedergegeben, die z. B. den Safety oder CEUS Guidelines u.a. der EFSUMB bzw. andere Institutionen zugrunde liegen. Ich kann daher nur jeder Kollegin oder Kollegen eine
Buchkopie als PDF oder für den eBook Reader empfehlen, um bei Interesse und Bedarf, die einzelnen gesicherten Erkenntnisse so kompakt nachlesen und parat haben zu können. Für mich ist dies ein essenzieller Klassiker, der bei einem Ultraschall-Anwender im neuerdings virtuellen Buchregal nicht fehlen sollte. Ein großer Dank an die Editorin Gail ter Haar daher für die Auswahl der renomierten Autoren, Themen und ihren Einsatz, um einen kostenlosen download zu ermöglichen!

\section{Christian Kollmann}

\section{THE SAFE USE OF ULTRASOUND IN MEDICAL DIAGNOSIS}

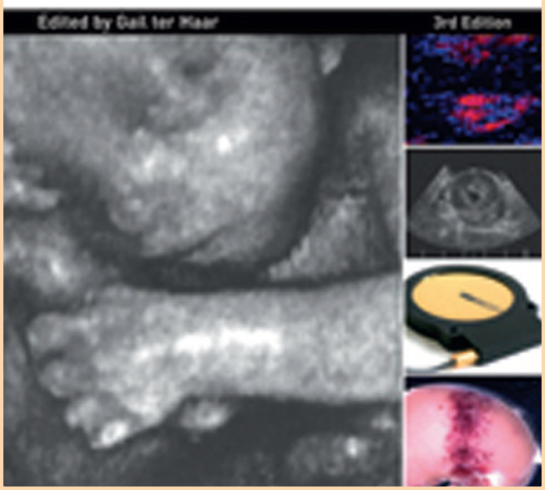

